Earth-trade unionists and managers alike. Ideas have moved not only out through society from their intellectual fountainhead, but outwards throughout the Commonwealth; and they are the same ideas, whether in Nigeria or Pakistan.

Writing fresh from the impact of this meeting, which developed an atmosphere of common effort which, in the Duke of Edinburgh's words, you could cut with a knife, I am bound to over-estimate the effects of this event. Yet that it was an event, with its own tiny place in the story of the Commonwealth, I am convinced. The members, when they return to the intrigues, the disappointments, the prejudices and the passion of real life in their countries (conferences are never real life) will carry back with them not only an experience of friendship-and that was abundantly evident-but also a reinforcement of faith and hope. Is anything of more value?

GuY Hunter

\title{
PHYSICS OF GAS FLOW AT VERY HIGH SPEEDS
}

$\mathrm{T}$ HE summer discussion meeting of the Physical Society was held in the University of Manchester during July 16-17 under the auspices of the Departments of Applied Mathematics and of the Mechanics of Fluids. The subject was "The Physics of Gas Flow at very High Speeds", that is to say, speeds so high that changes in kinetic energy are comparable with the energy required to produce interesting physical changes such as dissociation, ionization and substantial emission of radiation. The implications for the aerodynamics of man-made projectiles travelling at extreme speeds, and also of meteors, were included in the discussion. The shock tube, the principal research tool in this field, was discussed at length, both as regards design and instrumentation for use with very strong shock waves, and in connexion with the results so far discovered by its use. Prof. S. Devons, vice-president of the Society, presided over the meeting.

As Prof. M. J. Lighthill, of the Department of Mathematics, University of Manchester, said in his introductory remarks, the object of the meeting was to promote studies in a field where two great new scientific disciplines of the twentieth century meet and overlap. These disciplines are, on one hand, chemical kinetics, as grounded theoretically on the study of collision processes and experimentally on spectroscopy as interpreted by the quantum theory of radiative transitions; and on the other hand, fluid dynamics, as it has grown up since the discovery of the basic concepts of the boundary layer and the shock wave during the first decade of this century, using the wind-tunnel as the principal experimental tool. Neither subject had yet reached the dignified status of a science in the nineteenth century, when as Sir Cyril Hinshelwood has observed, chemical reactions were classified mainly into those that go and those that do not go, and when fluid dynamicists were divided into hydraulic engineers who observed things that could not be explained and mathematicians who explained things that could not be observed. In the great, and largely successful, struggles of the past fifty years to reach some sort of systematic understanding in both these two fields, the scientists involved were naturally disinclined to complicate their problems by introducing ideas from quite different disciplines. So until very recently it has been the rule for fluid dynamicists to know almost no physical chemistry, and physical chemists almost no fluid dynamics.

During the past five years, however, a movement has grown up with the object of making now advances by pooling the knowledge that has been acquired in these fields. To this end, fluid dynamicists have set themselves to learn about chemical kinetics and students of chemical kinetics have started to study fluid dynamics. These herculean labours have already begun to make important improvements in our knowledge of combustion processes, and a number of conferences have been held in this field. More recently still, external aerodynamics (that is, the study of flow around projectiles moving through the atmosphere) has reached the stage where this pooling of ideas is necessary. To mention an extreme example, it has been announced in the House of Commons that the Americans propose to shoot at Ascension Island from their missile-testing station in Florida. A simple computation of the elliptic orbit which such a missile would have to follow (after it has left the atmosphere and its rocket motors have burnt out) shows that it would have to re-enter the atmosphere with a speed of at least $6 \mathrm{~km}$. $/ \mathrm{sec}$. But a speed of half this would already be sufficient to cause considerable chemical changes in the air around a projectile. These changes would involve dissociation of oxygen and nitrogen molecules into free atoms, as well as the formation of nitric oxide. At speeds of $6 \mathrm{~km}$. $/ \mathrm{sec}$. the dissociation of oxygen near the front of the projectile would be nearly complete, and that of nitrogen well advanced. These changes may be expected to have important effects on the airflow about the body, and hence on the aerodynamic heating which is, of course, what constitutes the main difficulty of flight at these speeds. They constitute the main reason why aerodynamicists have been learning chemistry.

The reason for the converse process is the development of the shock tube, which physical chemists have found increasingly useful for studying fast gas reactions since Kantrowitz advocated its use for this purpose five years ago. Developed originally for studying the fluid dynamics of shock waves under conditions far more flexible than could be obtained in wind-tunnels, it has found additional use as a means of studying how reactions proceed in time after the pressure and the translational temperature of a gas mixture have been instantaneously raised by known amounts. For example, the shock tube has been used in this way to determine the rates of dissociation of iodine, bromine and nitrogen tetroxide. Since the shock tube is so useful both in this kind of work and in studying problems of very-high-speed aerodynamics, two of the five main lectures at this conference were devoted to it.

After giving this introduction Prof. Lighthill then proceeded to read his own lecture on the dynamics of a dissociating gas. He began by outlining the scientific advantages of starting, in such a difficult 
subject, by studying, both experimentally and theoretically, the behaviour of a pure diatomic gas capable of dissociation into free atoms. The theory falls naturally into three parts: equilibrium theory; quasi-equilibrium theory of the transport properties, including radiative transfer ; and flow theory involving large departures from equilibrium.

The first part includes the fundamental thermodynamics and statistical mechanics of the atommolecule mixture, and also the theory of the flow outside the boundary layer between a body flying at extreme speeds and the shock wave which travels ahead of it, in cases where equilibrium is reached behind this shock wave in a negligible time. Prof. Lighthill described the Busemann theory of this flow, in which the shock wave is wrapped around the body like a piece of paper around a parcel, and also the recent developments by Freeman and Chester in which second and third approximations to the flow pattern, based on Busemann's approach as a first approximation, have been found, yielding more accurate equations for the shape of the shock wave. $\mathrm{He}$ gave also an alternative theory of the flow near the stagnation point on a sphere, in which the only approximation used is the assumption of incompressible flow behind the shock wave. The good agreement between these two theories, based on the neglect of quite different features of the flow, in their region of overlap, gives one increased confidence in both.

Prof. Lighthill discussed briefly the kinetic theory of the diffusive heat transfer (stressing the importance of interdiffusion of atoms and molecules) and its application to the theory of the laminar boundarylayer near the front stagnation point on a bluff body, touched on the physical mechanisms of radiative transfer, and then turned to theories of flow involving large departures from equilibrium. Freeman had calculated the passage to equilibrium behind a uniform plane shock wave, an important feature of this process being the decrease in relaxation-rate as the translational temperature drops. He was now engaged in applying similar methods of calculation to the curved detached shock wave ahead of a projectile.

Mr. H. K. Zienkiewicz, of the Department of Mechanics of Fluids, University of Manchester, then gave a lecture on aerodynamics at extreme speeds. This was based on slides prepared to illustrate detailed flows which he had computed using tables of the composition and thermodynamic properties of air in dissociative equilibrium over a wide range of pressures and temperatures. $\mathrm{H}_{\theta}$ discussed plane shock waves and expansions in this way and various flows obtained by combining them, and ended by giving a theory for axisymmetrical flow around a cone. In all cases he made a comparison between the behaviour to be expected of air and that which would be predicted if changes in composition were neglected or if the specific heats remained. constant.

The lectures on design and instrumentation of shock tubes were given by members of the High Speed Section of the Aerodynamics Division of the National Physical Laboratory, Teddington. Dr. B. D. Henshall first spoke of the reasons for the popularity of the shock tube as a means for producing flows with high stagnation temperature (of the order of ten or twenty thousand degrees). He described the elementary theory of the shock tube, and its modifications by the effect of the walls, stressing the importance of wall resistance to the turbulent flow in the neighbourhood of the contact surface, with its somewhat surprising effect of decelerating the shock wave but accelerating the contact surface itself. He described the use of hypersonic nozzles at the downstream end of shock tubes to attain very high local Mach numbers at the expense of low Reynolds number, and pointed out that, if these nozzles are to 'start' satisfactorily, an initial back-pressure of a fraction of a micron of mercury is necessary. He described shock tubes using more than one diaphragm, and discussed the factors influencing diaphragm design.

Dr. Henshall then described in detail the proposed 6 in. square shock tube at the National Physical Laboratory, and discussed how different aspects of problems of free flight at extreme speeds can be simulated, using the straight portion of the shock tube or the flow after one or two subsequent expansions. Dr. O. L. Schultz described the timing and triggering devices which will be used in this shock tube, the pressure and temperature gauges which are at present proposed, and the way in which the latter will be used to measure transient heat transfer rate. $\mathrm{He}$ also described a method of inferring overall drag by measuring the acceleration of a freely floating model.

In the subsequent discussion Mr. C. H. E. Warren, Royal Aircraft Establishment, Farnborough, described the design of the shock tube under construction there. He was against the use of multiple diaphragms and favoured the straightforward use of hydrogen as a driver, raised to high temperature and pressure by combustion with oxygen. He agreed with Dr. Henshall that valuable work on simulating flight at extreme speeds could be done if the stagnation temperature and Reynolds number were reproduced accurately, even though the Mach number in the exporiment was far too low. Inaccuracy of Mach number might be got over by changing the geometry.

Mr. E. M. Dowlen, of the English Electric Co., Ltd., Luton, then described the parallel work going on in his laboratory. He stressed the convenience of using small tubes (for example, of 2 in. diameter), and agreed with workers at Teddington on the advantages of surplus gun-barrels as the material of the highpressure sections. Later, Dr. R. Hide, of the Atomic Energy Research Establishment, Harwell, described the shock tubes in use there, and the work going on concerning the application of magnetic fields to the highly ionized gas behind a very strong shock wave.

A different aspect of gas flow at very high speeds was discussed in the lecture by Dr. J. G. Davies, of the Jodrell Bank Experimental Station, Manchester University. After a general account of the astronomy of meteors, he described the techniques for observing, visually, photographically and by radio echoes from their ionized trails, the entry of meteors into the atmosphere at speeds between 11 and $72 \mathrm{~km}$. $/ \mathrm{sec}$. He mentioned the importance of observations of the drift of meteor trails as a source of information on winds in the upper atmosphere.

Dr. Davies pointed out that almost all meteors are less than a centimetre in diameter and evaporate completely (owing to heat transfer by direct collision with atoms and molecules of the atmosphere) at altitudes where the mean free path is large compared with the diameter. He described a theory of this process, from which a theoretical expression for the variation of light intensity with distance along the meteor trail can be determined. In some cases this 
No. 4529 August 18, 1956

theory is inapplicable because fragmentation of the meteor occurs, leading to greatly increased radiation. and quicker evaporation. Bodies large enough to enter the denser region of the atmosphere, where they acquire a compressed-air cap ahead of them which largely shields them from the direct blows of atmospheric molecules, are those producing trails much brighter than the brightest stars, and they are observed far too rarely for any systematic knowledge of their aerodynamics to have been found. The larger ones reach the Earth in the form of meteorites, which are mostly of irregular shape, often pitted with deep, roughly spherical holes. Prolonged discussion at the meeting of whether information concerning the aerodynamical experiences of these bodies can be found led to no very helpful conclusions.

The last lecture was by Dr. Wayland Griffith, of the Palmer Physical Laboratory, Princeton University, where much of the pioneering work with shock tubes was done under the direction of Dr. Walker Bleakney. Dr. Griffith has been studying in Britain for the past year. He described fundamental work on the experimental verification of the RankineHugoniot equations for a shock wave passing through various gases, using their known equilibrium thermodynamies. This work shows, for example, that although the transverse vibrations of the carbon dioxide molecule are excited in such experiments with a relaxation time equal to that found from acoustic attenuation work, the longitudinal (valence) vibrations still remained unexcited after a time long compared with this (of the order of milliseconds).

Dr. Griffith discussed experimental determinations in shock tubes, by Davidson, Hornig and others, of dissociation rates, and gave theories, both of the chemical kinetics of dissociation, and of the fluid dynamics of the process which may be postulated to infer the rates from the experimental observations. He then described the work which had been done in Dr. A. R. Kantrowitz's laboratory at Cornell University on ionization produced by strong shock waves in argon. The observed time-delays before equilibrium ionization has suggested improved approaches to the kinetics of the ionization process. The luminosity of the shock front itself, which has sometimes been observed, is the result of impurities. The measurements of conductivity of a gas with high electron density has shown satisfactory agreement with theory.

In his concluding address Prof. Devons remarked on the impressive degree of collaboration between applied mathematicians, physicists, chemists and engineers which seems already to have taken place in this field. He emphasized, however, that an even greater degree of co-operation will be necessary, both if the maximum effectiveness for man-made projectiles travelling at extreme speeds is to be achieved, and if the shock tube is to be fully utilized for uncovering the secrets of high-speed gas reactions.

\section{THE WEIZMANN INSTITUTE OF SCIENCE}

$\mathrm{T}$ O mark the tenth anniversary of the foundation of the Weizmann Institute of Science, the Board of Governors, together with representatives of supporting groups in Great Britain, the United States, Canada and Switzerland, held a conference in London early in July.

The Weizmann Institute had its origin in the Daniel Sieff Research Institute at Rehovoth, a town about twelve miles south of Tel Aviv. The Sieff Research Institute had been set up in 1934 by $\mathrm{Mr}$. and Mrs. Israel N. Sieff, of London, as a memorial for their youngest son, who had died the year before. Dr. Chaim Weizmann (1874-1952), with whom $\mathrm{Mr}$. and Mrs. Sieff consulted before founding the Institute, assumed its direction and was responsible for its first scientific programme.

In 1944 a group of Dr. Weizmann's friends in the United States launched a project to mark his seven tieth birthday and raised five million dollars for the purpose. It was then decided to establish the Weizmann Institute of Science, the Daniel Sieff Research Institute being retained as the parent unit.

The foundation stone for the expanded establishment was laid on June 3, 1946, and the Weizmann Institute was formally inaugurated and began functioning in November 1949. The Institute now covers an area of some ninety acres, with four large buildings in addition to the parent structure, the Sieff Institute, and an Institute of Physics in construction. Compared with the Sieff Institute's original staff of ten scientists and some twenty technical and administrative workers, there are now 130 scientists and more than 350 other personnel.
The recent conference was divided into five sessions. Two of these sessions were under the chairmanship respectively of Sir Francis Simon and Sir Solly Zuckerman, and dealt with the Institute's scientific interests.

The Institute engages primarily in research and has no teaching responsibilities, apart from the affiliation of several of its departmental heads to the Hebrew University in Jerusalem as visiting professors. Its main research interests are in the exact sciencesmathematics, physics, nearly all branches of chemistry, and some branches of biology. The desire has lately been expressed by scientific staff at various levels for the introduction of postgraduate teaching as a means of augmenting Israel's reservoir of trained professional man-power and also as a stimulus to their own work. As a result of a recommendation of a Committee which was set up under the chairmanship of Sir Ben Lockspeiser, the Board of Governors has now decided to provide more opportunities for postgraduate training than were available in the past. This Committee also recommended "that the provision of special courses as a useful service for the country, as, for example, in the fields of radio chemistry, plastics chemistry, electronic computing and numerical analysis, be encouraged within the capacity of the Institute". Some time ago the U.S. Government decided to include support of the Radioisotope School at the Weizmann Institute, for training professional men in the use of isotopes, as part of a series of grants it was making for higher learning and research in Israel.

In addition to the heads of departments of the Institute who came from Israel, the conference was 